\title{
ARTIFICIAL INTELLIGENCE AS A SERVICE IN DISTRIBUTED MULTI ACCESS EDGE COMPUTING ON 5G EXTRACTING DATA USING IOT AND INCLUDING AR/VR FOR REAL-TIME REPORTING
}

\author{
Pavan Madduru \\ Email:pavan.telco@gmail.com
}

\begin{abstract}
To meet the growing demand for mobile data traffic and the stringent requirements for Internet of Things (IoT) applications in emerging cities such as smart cities, healthcare, augmented / virtual reality (AR / $V R)$, fifth-generation assistive technologies generation (5G) Suggest and use on the web. As a major emerging $5 G$ technology and a major driver of the Internet of Things, Multiple Access Edge Computing (MEC), which integrates telecommunications and IT services, provides cloud computing capabilities at the edge of an access network. wireless (RAN). By providing maximum compute and storage resources, MEC can reduce enduser latency. Therefore, in this article we will take a closer look at 5 G MEC and the Internet of Things. Analyze the main functions of MEC in $5 G$ and IoT environments. It offers several core technologies that enable the use of MEC in 5G and IoT, such as cloud computing, SDN / NFV, information-oriented networks, virtual machines (VMs) and containers, smart devices, shared networks and computing offload. This article also provides an overview of $M_{E C}$ 's role in $5 G$ and IoT, a detailed introduction to MEC-enabled $5 G$ and IoT applications, and future perspectives for MEC integration with $5 G$ and IoT. Additionally, this article will take a closer look at the $M E C$ research challenges and unresolved issues around $5 G$ and the Internet of Things. Finally, we propose a use case that MEC uses to obtain advanced intelligence in IoT scenarios.
\end{abstract}

KEYWORDS:Multi-access edge computing, mobile edge computing, MEC, 5G, Internet of things, IoT, low latency, edge computing, edge intelligence.

\section{Introduction}

2019 ushered in the era of the fifth generation (5G) wireless network. Compared with $1 \mathrm{G}$ to $4 \mathrm{G}$, the role of $5 \mathrm{G}$ is more diverse. Its purpose is to support the rapid development of the Internet and ICT. It has the following two aspects: (i) 5G must support communication when in use; management, data processing and content distribution (4C); (ii) In 5G applications, it supports several new applications that require unprecedented latency and high access speed, such as mobile games, virtual / augmented reality (AR / VR) and factory automation.

The Internet of Things (IoT) has grown steadily in recent years, allowing many devices with limited resources to communicate over the Internet. With the help of the Internet of Things, the rapid growth of many mobile devices (eg smart devices, clothing, smartphones) is providing a wide range of information. Cisco estimates that computing hardware, IoT devices, and people will exceed 500 Zeta bytes (ZB) by 2020. However, many IoT tools lack planning, storage, and communication resources. For example, the processing and storage capacity of a machine is low, so they use the cloud to hone their skills.

In recent decades, cloud services have also become a new kind of computing. As a typical cloud computing model, Mobile Cloud Computing (MCC) is being implemented as a promising solution that includes self-awareness, availability, scalability, and adaptability. Although the MCC has made many services available, especially applications that run on mobile devices (phones, tablets, etc.), it cannot meet the strict requirements of $5 \mathrm{G}$ and IoT (such as low latency). The reasons for this are as follows: (i) First, due to the long response time, a centralized cloud architecture will affect the quality of the end user experience (QoE); (ii) Second, as all information must be sent to the central cloud, there is still leaks and privacy data in the central MCC; (iii) Third, most IoT data is traditionally processed through a central cloud connection, data latency, and network bandwidth. Therefore, there is consensus that MCC alone will not be sufficient to support 5G and IoT communication and computing, the popularization of 5G and IoT, AR / VR, healthcare, smart transportation, location services and other emerging applications. , MCC and other new features.

\section{The Birth of MEC and Its Advantages}

Due to the disadvantages of MCC, the concept of edge cloud was proposed. The cloud concept was presented by Satyanarayana et al., Which is a midtier three-tier model consisting of a centralised data centre, an edge cloud platform (i.e., a cloud), and a terminal. The cloud, which is the original form of the edge cloud, can make it easier for end-users to benefit from computing and storage space. With the cloud 
service, the services of nearby remote server centres can be extended to end-users. However, the Cloudlet has its limitations: The Cloudlet can only be accessed by WiFi access points that can only cover small areas, so ubiquitous computing cannot be provided.

To address the above challenges, the European Telecommunications Standards Institute (ETSI) recently launched in 2014 a new architecture, dubbed the Mobile Edge Computing (MEC) concept. MEC is a unique platform that can be deployed while providing services and IT capabilities over RAN radio communications close to mobile users [8]. The first MEC definition refers to the use of bases (BSs) to evaluate the performance obtained from the data. Finally, in December 2014, ETSI launched the MEC Industry Integration Group (ISG) by launching the MEC model to promote and accelerate peripherals' development while operating on mobile devices. By enabling environmental opportunities for multiselling vehicles at the end of the RAN, ETSI MEC ISG achieves the goal of overcoming the challenges faced automatically while testing services to ensure high speeds and latencies [9]. Since September 2016, the term "Telephone" has been removed from MEC to expand MEC's use across multiple networks (such as fixed technologies, WiFi, etc.), with ETEC ISG selected MEC Multi-use Edge Computing.

MEC, which identifies services during the RAN end (for example, BSs), makes the RAN end near the end of IT users and while computing competently, it achieves the goal of delivering low latency highbandwidth environments and access to radio communications. Due to its excellent features such as proximity/location/travel alerts, real-time light information radio information, high bandwidth, and low latency, Etc., MEC can allow many types of applications and services, especially those timesensitive applications like mobile AR / VR are a common feature of $5 \mathrm{G}$ and IoT applications. MECs can also be useful for service providers, as they can use MECs to gather more important information about people, interests, interests, and behaviours and improve more Customer Service (QoS) and QoE users accordingly. Also, the MEC model promises to offer a more user-friendly experience and enable more variety of IoT applications and is therefore recognised as a critical technology capability that has built an efficient way to identify $5 \mathrm{G}$ as well as IoT.

Comparison to Existing Surveys and the Contributions

There is much work to be done now, including MEC research in the field of technology or case studies first demonstrated the feasibility of standard and standard MEC applications. After this emphasised the integration of MEC and $5 \mathrm{G}$ and beyond technologies

${ }^{1}$ Rashid and Abd Razak., 2019

${ }^{2}$ Velev et al., 2018 including non-orthogonal multiplexing (NOMA), training tools, auxiliary aircraft (UAVs), electric radios and power plants, among others have extensively researched literature using traditional games including traditional games and growth in social media. With a focus on the application of MEC principles and the concept of games specifically provide a broader overview of the use of MEC technology for the implementation of IoT applications, and their synergies launched a study in MEC and refined key enabling technologies, terminology selection options, visualisation areas, and current settings, and more. MEC extension machines are categorised into methods for setting uploads and strategies for caching.

The tasks above focus on integrating 5G into MEC without light data or highlighting the game used in MEC applications without mentioning $5 \mathrm{G}$ or IoT or just demonstrating the integration of IoT and MEC without considering $5 \mathrm{G}$ or using a smart bank or focusing on the MEC itself without considering $5 \mathrm{G}$ or IoT or smart devices, or thinking only of devices that improve MEC devices, is very different from the 5G-focused work and research on IoT and MEC intelligent technology. Therefore, the work is new in the sense that we have done a detailed analysis of MEC towards intelligence for $5 \mathrm{G}$ and IoT. The main features of this document are as follows.

We detail the features and critical enabling technologies when using MEC in 5G and IoT.

- In particular, we are developing MEC's role in 5G and IoT, MEC enabled 5G and IoT applications and possible research guidelines for integrating MEC into 5G and IoT, respectively.

- We will also discuss the challenges and open issues of the MEC face now and in the future 5G and IoT, e.g., edge intelligence.

- Also, we proposed a particular use case for MEC helps to understand intelligence in IoT scenarios.

MEC (Multiple Access Edge Computing) is a hotly debated issue today. It is undoubtedly changing the way information is processed and supplied by different devices around the world ${ }^{1}$. Much of the hype caused by new technologies is related to its use cases. It is instrumental when combined with other dynamic and powerful technologies such as artificial intelligence (AI) and 5G, high-speed communication technologies ${ }^{2}$. These technologies enable multispace international computer systems to frame and improve real-time software such as self-driving vehicles, robotics, photography, details, and more ${ }^{3}$. While the goals of face recognition, when first 
created, determine the bandwidth for drugs that travel long distances due to the data generated by the development of the Internet of Things, the real-time growth is in the work model. will lead technology forward ${ }^{4}$.

\section{Multi-Access Edge Computing}

Multi-Access Edge computing is a term used for a cloud-based IT service environment located at the edge of the network ${ }^{5}$. It is the next step in the delivery of information and information processing to the customer. In 2016, the European Telecommunications Standards Institute (ETSI) changed this technology to its current name because of its use outside of mobile communications. Multifactor computing brings information closer to the collection point than expected to a central location thousands of miles away ${ }^{6}$. This has now been done to ensure that real-time information has no latency issues affecting job performance.Companies can also be encouraged to significantly reduce costs by implementing a section of the website, which reduces the amount of information needed during the cloud process. Developed in response to the growing number of IoT devices connected to the Internet, not only to receive data from the cloud but also to return it to the cloud ${ }^{7}$.

Although a single data generator can translate quickly and easily over the web, it can be a problem as multiple devices send data at the same time ${ }^{8}$. It also causes poor performance due to late payment and high-cost savings ${ }^{9}$. Multiple Access Edge Computing helps solve this problem because it is the place to store and operate things for these programs. In addition to general processing information, it also cleans the data and sends the relevant information to the cloud ${ }^{10}$.

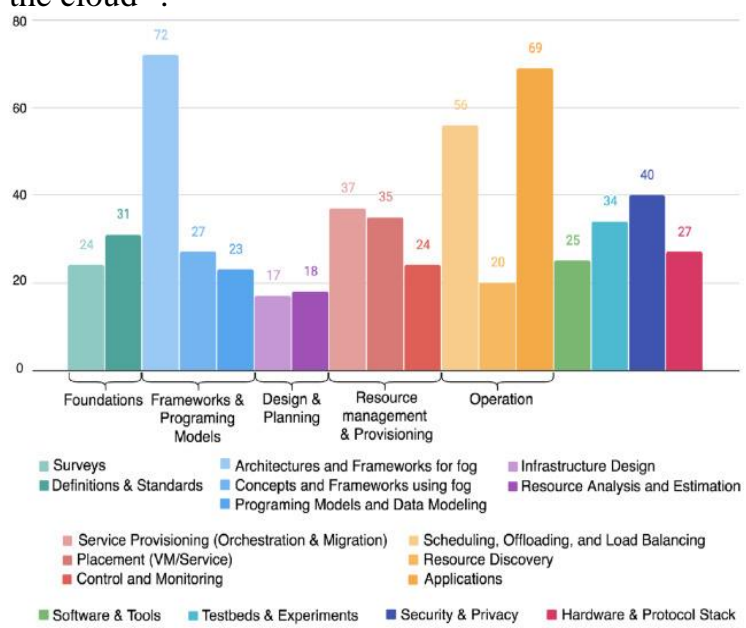

\footnotetext{
${ }^{4}$ Sharma et al., 2018

${ }^{5}$ Zikria et al., 2018

${ }^{6}$ Asif et al., 2020

${ }^{7}$ Monge et al., 2018
}

(Source: Ahmed et al., 2019)

Comparison with MCC

MEC can be considered as an evolution of MCC. There are significant differences between MEC and MCC. As shown in Table I, the differences between them are compared, and the MEC exceeds the ratio of MCC latency, energy savings, context-aware computing, privacy and security enhancements, Etc. 1) Low delay: The delay usually consists of propagation and transmission delay, queuing delay and processing delay, which depends on the matching distance, data rate and calculation. First, the propagation distance of the MEC is much shorter than that of the MCC, so the propagation delay of the MEC is much shorter than that of the MCC. In the edge network, communication is limited. Third, to handle the delay, the MCC has a large computing capacity, often several orders of magnitude larger than the edge devices. However, it should be noted that many users share a computational resource in an MCC environment, so from this perspective, the processing delay between the MCC and the MEC can be compensated. Overall, current studies, such as the total MCC delay, range from 30 to $100 \mathrm{~ms}$, which is unacceptable in time-critical $5 \mathrm{G}$ or IoT applications such as stand-alone driving, real-time online gaming, and high-quality VR / AR that require such a short delay more than $1 \mathrm{~ms}$. Unlike MCC, MEC is a promising technology capable of implementing touch-level latency for time-critical $5 \mathrm{G}$ and IoT services thanks to its short distance and simple protocols.

2) Energy-saving: IoT devices can limit battery capacity, but are designed to perform monitoring and regulate activities such as health monitoring, human identification and monitoring. Existing, batteries in IoT devices cannot be updated frequently and quickly. Fortunately, MEC can help streamline the use of IoT tools by bringing down the essentials from these IoT tools to the MEC server but satisfying the need too late and reliable. For example, experiments show that IoT devices' battery life in AR applications can be increased by $30-50 \%$ with the proposed resolution. In comparison, due to the distance between IoT devices and international computer organisations and the high availability of communication limitations, MCC is unable to help save energy and meet latency needs when setting up task scheduling and cheap service.

3) Data-alert: By using proximity to device-based devices, MEC can track users' information (e.g., location, speed, behaviour, Etc.) in real-time. Using that information, the MEC can provide specialised services to end-users, which is also a common feature of the MEC compared to the MCC. For example,

\footnotetext{
${ }^{8}$ Sk and Masilamani., 2018

${ }^{9}$ Nieto et al., 2019

${ }^{10}$ Ahmed et al., 2019
} 
retailers can use MEC staff to quickly respond to market conditions and customer needs based on customer information integration. For another example, intelligent farming can be obtained by digging a classification system near MEC 'services based on data collection on growing plants rules and prices. A third model, for tourists, MEC can support the use of software-controlled systems and AR/VR services to recommend user-defined data centres.

4) Confidentiality and Security Improvement: Compared to MCC, MEC has another attractive feature, i.e., confidentiality and improved security. For MCC: First, cloud computing plate- forms of MCC such as Microsoft Azure are large pieces of social media that are vulnerable to attack due to their high nature; Second, in the MCC, the ownership and legalisation of personal information is abandoned, resulting in private information leakage and loss. In comparison, MEC distributions are distributed and small, use personal information, and rarely attack the MEC. Also, MEC 'services can serve as a private cloud, simplifying the issue of information leakage. Therefore, MEC can benefit those services that require adjusting data exchange between services and end-users. An example of the development of the MEC in situations that do not require the provision of complex information to the centres in question is due to the conditions of all things being regulated and classified.

\section{Multi-Access Edge Computing Use Cases}

As part of a new partnership between Vodafone and the continent, the world leader in the automotive industry, one tests the use of different spacecraft (MEC) to enable new types of intelligence-based counterfeit car applications ${ }^{11}$. The project is part of a broader joint venture launch in the summer of 2018 to increase road safety and reduce six of the potential risks and pitfalls of technologies such as MEC, 5G and mobile phones for all $(\mathrm{C}-\mathrm{V} 2 \mathrm{X})^{12}$. The rationale for using the case stems from the desire to reduce accidents for pedestrians, cyclists and road users (such as children or adults) by identifying potential hazards shortly still ${ }^{13}$. It is especially true today (in busy cities, for example) and the increasing importance and emergence of independent drivers. From a practical point of view, the standard application is a combination of vehicle camcorder sensor information and Continental research and development pioneering intelligent design ${ }^{14}$.

The purpose of the application smart application is to study close to real-time video streaming from the

\footnotetext{
${ }^{11}$ Condoluci et al., 2018

${ }^{12}$ Rezvy et al., 2019

${ }^{13}$ condo and Jun 2019

${ }^{14}$ Sodhro et al., 2018

${ }^{15}$ Lin et al., 2016

${ }^{16}$ Zafeiropoulos et al., 2020

${ }^{17}$ Javaid et al., 2018
}

front-facing camera, for example, in a vehicle ${ }^{15}$. He then decides whether a dangerous situation arises or is likely to occur (for example, children may cross the street). Today's complex and computationally powerful learning algorithms drive these types of intelligent programming. The question arises as to where these algorithms are used ${ }^{16}$. Now, MEC is taking over the responsibility of new telecommunications technologies. The MEC development for communications networks has mostly overshadowed the implementation of distribution while developing extensive, potentially deep/remote services, including radio communications ${ }^{17}$. The MEC concept can be used on both $4 \mathrm{G}$ and $5 \mathrm{G}$ networks as it is not dependent on the type of Office radio used. The image below illustrates this. The information stream was sent from the vehicle via $4 \mathrm{G}$ or $5 \mathrm{G}$ at the top of the website, which makes it easy for experts to interpret the messages in real-time ${ }^{18}$.

The MEC also allows non-telecommunications companies, such as car companies, to carry out heavy work on the mobile network. It generates several positive aspects centred on this research and communication test ${ }^{19}$. First, MEC offers the opportunity to improve real-time operations and accelerate real-time application development compared to shared hosting in the central region area $^{20}$. Because MEC stands for Mobile Network Cloud Service Platforms, it can host programs such as conducting intelligence car applications ${ }^{21}$. The typical response is essential to independent drivers for accident prevention, especially the vehicle's high speed and a large number of unintentional or unregulated activities of road users, such as the walkers. MEC provides a low latency by classifying vehicle data from vehicle network directors near MEC locations in the telecommunications network ${ }^{22}$. Second, MEC provides motor vehicles and engineers the best choice in deciding where to do massive computing or not, where to integrate data of multiple sensor compounds (also called sensor fusions), and how to build the range Service Design ${ }^{23}$.

With the help of the Telco network's large cloud compartments, individuals planning tasks by engineers who will be assigned to the vehicle can now be transferred to the nearest cloud data centre ${ }^{24}$. Similarly, some parts of the application will be in the centre while it is now possible to approach vehicles

\footnotetext{
${ }^{18}$ Rashid and Abd Razak., 2019

${ }^{19}$ Velev et al., 2018

${ }^{20}$ Velev et al., 2018

${ }^{21}$ Alsaeedy and Chong., 2019

${ }^{22}$ Sharma et al., 2018

${ }^{23}$ Adeel et al., 2018

${ }^{24}$ Zikria et al., 2018
} 
to improve response times ${ }^{25}$. Third, MEC increases cost-effective application performance, resulting in lower unit costs for network communication, in this automated situation ${ }^{26}$. Modern learning programs benefit from high-speed machines ${ }^{27}$. Software chips, such as graphics production units (GPUs), have long been proven to be suitable for high-performance gaming consoles. Still, over the years, they have also been shown to increase machine learning devices' performance through high throughput. (Sk ma Masilamani., 2018). However, these high-end viewing processors are costly and include a fastpaced cycle of new technologies ${ }^{28}$. Thus, by putting smart information on the communication line, the application can use the much faster tools because it is available on the web and it can be used as other clouds $^{29}$.

The disadvantage is that most high-end machine tools in the car reduce costs. It also prevents mechanical devices from slowing down the lifespan of the vehicle $^{30}$. Fourth, the shared cloud edge service model assumes that network connections, such as vehicles in a single part of the cloud, share the same cloud resources ${ }^{31}$. This economic growth is because the same specialised equipment can serve many vehicles, not just one. It also results in lower energy consumption in the vehicle and lower costs for all vehicles involved ${ }^{32}$.

In conclusion, if an essential part of the model is that the hosting staff has integrated on the integrated platform in the mobile and external network located in the middle of the cloud ${ }^{33}$. It can significantly reduce the country to a share as the return of total traffic, which is essential in the future ${ }^{34}$. Researchers predict ExaBytes data for the month until 2025 for connected vehicles only ${ }^{35}$.

The team project included Continental (building the new application) and implementation teams Vodafone, Juniper, Saguna, Xantaro and Netrounds. The team directly installed MEC and cloud computing at an automotive test site at the Aldenhoven test site in Germany ${ }^{36}$, where Vodafone's $5 \mathrm{G} \mathrm{lab}^{37}$. The cloud-based cloud includes Saguna Networks' MEC software, communications

\footnotetext{
${ }^{25}$ Asif et al., 2020

${ }^{26}$ Hayes and Omar., 2019

${ }^{27}$ Monge et al., 2018

${ }^{28}$ Ahmed et al., 2019

${ }^{29}$ Nieto et al., 2019

${ }^{30}$ Condoluci et al., 2018

${ }^{31}$ Rezvy et al., 2019

${ }^{32}$ Chu and Jun 2019

${ }^{33}$ Mohanta et al., 2019

${ }^{34}$ Sodhro et al., 2018

${ }^{35}$ Lin et al., 2016

${ }^{36}$ Mohanta et al., 2019
}

management and virtualisedIPSecurity gateways powered by Juniper software ${ }^{38}$. Netroundsvirtualised software and active sensors, and a Vodafone cloud supported foundation while developing and hardware acceleration and production scene. After the project team implemented the AI application at the end of the day, several tests and support from Xantaro were conducted $^{39}$. It allows Continental to analyse the capabilities of a highly specialised application in dealing with changing radio conditions, cell load cell radios, and vehicle speeds, for example, with the Vodafone team investigating the performance of an end-to-end regulatory framework ${ }^{40}$.

Using different technologies, many organisations and companies in various business and commerce areas are looking to improve their operations and find new ways to improve their performance look online and with customers ${ }^{41}$. However, some case studies of multipurpose edge calculations and their advantages are listed below ${ }^{42}$.

\section{Edge Intelligence (EI)}

Edge intelligence (EI), based on technological knowhow (for example, machine learning, deep neural network, Etc.), has already been seen as one of the critical elements in 5G networks and seems to be an essential performance component for future $6 \mathrm{G}$ networks to sustain operations, innovation and new services ${ }^{43}$. As such, the purpose of this White Paper is to provide a clearer understanding of why light is an essential aspect of $6 \mathrm{G}^{44}$, and what design principles and advanced technologies front help in building the smart end for $6 \mathrm{G}^{45}$. In recent years, we have seen the growth of the market and the adoption of intelligent solutions in a wide variety of ICT applications. The increased awareness of the service areas in such diverse fields, including with the experts to help one, and video / audio recording ${ }^{46}$, knowledge of the town and drive themselves. The entire industry is developing new forms - Industry 4.0 is a great model that aims to number the manufacturing, robotics, automation and communications industries as part of the changing numbers ${ }^{47}$.

\footnotetext{
${ }^{37}$ Hossain and Muhammad., 2017

${ }^{38}$ Trakadas et al., 2019

${ }^{39}$ Chen et al., 2016

${ }^{40}$ Vukobratovic et al., 2016

${ }^{41}$ Javaid et al., 2018

${ }^{42}$ Zafeiropoulos et al., 2020

${ }^{43}$ Rashid and Abd Razak., 2019

${ }^{44}$ Velev et al., 2018

${ }^{45}$ Alsaeedy and Chong., 2019

${ }^{46}$ Sharma et al., 2018

${ }^{47}$ Adeel et al., 2018
} 
Also, increasing use of computers and software requires new types of fraudulent applications, such as power and time limits for data and data transfer, and privacy and security ${ }^{48}$. Interest in specialist information resulting from development recently, high performance and cost-effective planning, and the increased number of data produced by machines of different locations, from the mobile experience of individual businesses doing business ${ }^{49}$. Efficient, low-cost operation and storage for computer collection are available for anyone on a credit card, and they much satisfy the need for sophisticated simulation to process a large amount of information $^{50}$. The highest place-based site offering (the height of equipment) is being established for the top and the IT design ${ }^{51}$. Devices to generate and delete data stand at the edge of the network, close to users and systems that monitor, control, or control ${ }^{52}$. However, very little attention has been paid to this megatrend. Smart Internet terminals, devices, and mobile numbers ${ }^{53}$, Things (IoT), a large-scale retailer, as well as sensor and video cameras, are increasing the number of generates hundreds of ZBinformation at the end of the website ${ }^{54}$. Also, increasing the use of small-scale memory modelling tools such as TinyML is a significant contribution. Considering this, experimental design means that the integration of the experimental cloud is expanding towards the border ${ }^{55}$. Edge computing (EC) is a specialised type of cloud computing that performs several operations and stores data related to specific services from the central cloud to the most complicated web site and is well suited to company data and end-users ${ }^{56}$. The beneficial benefits of using scripts over the existing $5 \mathrm{G}$ networks, including improved performance, improved routes, and new services are deficient ${ }^{57}$. Smart Edge in $6 \mathrm{G}$ makes a massive contribution to all of these critical aspects above. Also, competence is the key to enabling the development of a whole new class of products and services $^{58}$.

New opportunities and business and innovations in peripheral computers and the lightest edge may soon appear in many areas ${ }^{59}$. The term " AI read" is also sometimes used to emphasise, not only to work on the border but also to use computers between the edge

\footnotetext{
${ }^{48}$ Zikria et al., 2018

${ }^{49}$ Asif et al., 2020

${ }^{50}$ Hayes and Omar., 2019

${ }^{51}$ Sk and Masilamani., 2018

${ }^{52}$ Monge et al., 2018

${ }^{53}$ Nieto et al., 2019

${ }^{54}$ Ahmed et al., 2019

${ }^{55}$ Condoluci et al., 2018

${ }^{56}$ Rezvy et al., 2019

${ }^{57}$ Chu and Jun 2019

${ }^{58}$ Sodhro et al., 2018
}

and the centre ${ }^{60}$. Although the definition at the end and AI computing are different, with a slight difference, the terms used similarly refer to the adaptation of actions that take place on computers outside the central cloud ${ }^{61}$. One definition of $5 \mathrm{G}$ internal and external communication technologies comes from ETSI Multi-Access Edge Computing (MEC) efforts. In this architectural design, the host uses the top-level to facilitate the use of software and services at the end ${ }^{62}$. The ETSI MEC platform binds MEC applications and services to the cell room via API encryption, such as accessing database information bases and supporting shared communications ${ }^{63}$.

From a research study, knowledge about the analysis of information and the development of responses to or near the website is obtained by data and subsequent ${ }^{64}$. Thus, knowledge can reduce waiting time, cost, and security, thus increasing the business's efficiency. From a visual point of view, the smartest point refers directly to the smart services and actions performed at the end of the website ${ }^{65}$, perhaps in the user group, in the customer segment, or close to the user or customer, or beyond the boundary lines ${ }^{66}$. Borderline management, at its core, involves increasing the level of information processing and the ability to filter information to the general public. However, intelligence has been described as "a priori"67. With the development of borderline expertise, it is possible to bring a variety of exercise materials to both groups as well as collections to enable them to learn and share what they have learned and other (borderline) networks that provide valueadded services or efficiency ${ }^{68}$.

Thus, it can be predicted that the development of networks towards $6 \mathrm{G}$ will take into account the efficient distribution of intelligent signals to change the light from cheap while in peripheral computing resources ${ }^{69}$. Targeted settings include advanced Internet of Things applications and number change functions. Also, a global knowledge needs to be understood by standard intelligent systems, mostly provided by the state of the machine and human interaction (e.g., in the work environment) for

\footnotetext{
${ }^{59}$ Lin et al., 2016

${ }^{60}$ Mohanta et al., 2019

${ }^{61}$ Trakadas et al., 2019

${ }^{62}$ Vukobratovic et al., 2016

${ }^{63}$ Chen et al., 2016

${ }^{64}$ Hossain and Muhammad., 2017

${ }^{65}$ Zafeiropoulos et al., 2020

${ }^{66}$ Javaid et al., 2018

${ }^{67}$ Rashid and Abd Razak., 2019

${ }^{68}$ Velev et al., 2018

${ }^{69}$ Alsaeedy and Chong., 2019
} 
security reasons ${ }^{70}$. In the meantime, for oheisälyä upgrade software and tools already in their baby, and we will see the edge, like Coral2 and Jetson3, who can implement smart data design. However, existing solutions that provide the intelligence required for property and energy, also have time ${ }^{71}$. Many widely used machine learning and deep neural network algorithms are still relied upon as appropriate algebra transistors to perform a wide range of numerical calculations on set data ${ }^{72}$. In the future, the number and availability of information space will only increase, but AI implementation requirements will increase the difficulty for determining (near) ultralow real-time latency applications. we note that this condition may not be sustainable in the long run ${ }^{73}$. In providing an excellent example of the inadequate tools and software in 5G, we remember that in regular operation, especially deep neural networks (DNNs). The higher levels they learn to add conditions high performance by providing benefits and is sometimes shortened to low status ${ }^{74}$. The advent of chipset technology is not as fast-growing as smart devices to meet market needs and demands ${ }^{75}$. Nanophotonic methods can help in this regard: DNN motions are usually large matrix, and Nanophotonic circles can perform these movements at almost the speed of light and are optimal due to photons' nature. Simple, photonic/optical data processing devices using electromagnetic radiation (such as laser beams) to store, transmit, and process data ${ }^{76}$. Optics has been used for many years, but to date, it has been limited to laser radiation on fibre ${ }^{77}$. Nanophotonic technologies that use signals to classify and store information can increase the comparison of highthroughput intelligence about latency, bandwidth, and workforce ${ }^{78}$.

Mental retrieval is a meaningful way to eliminate bottlenecks in translating information between devices and memories into the mechanical system ${ }^{79}$. Internal memory stimulated by the observation of the transfer of data from small cells to memory in the back-and-forth machine (through small lines ${ }^{80}$ ), communication memory is an essential function and bottleneck in software records. Attempts to combine thoughts and memories are referred to differently in literature such as mindfulness, classification of

\footnotetext{
${ }^{70}$ Sharma et al., 2018

${ }^{71}$ Adeel et al., 2018

${ }^{72}$ Zikria et al., 2018

${ }^{73}$ Asif et al., 2020

${ }^{74}$ Hayes and Omar., 2019

${ }^{75}$ Monge et al., 2018

${ }^{76}$ Sk and Masilamani., 2018

${ }^{77}$ Nieto et al., 2019

${ }^{78}$ Ahmed et al., 2019

${ }^{79}$ Rezvy et al., 2019

${ }^{80}$ Nieto et al., 2019
}

memories, and movement of memories ${ }^{81}$. These efforts can be divided into two parts: moving ideas over memory or planning closer to memory, and making classifications on what to organise or what one do remember. Internal memory seems to be an appropriate response to support the speed of DNN devices $^{82}$. The system on home chips, such as the Adaptive Computing Acceleration Platform (ACAP), is another way to use intelligence ${ }^{83}$. ACAP combines broad-spectrum functionalities with different $\mathrm{AI}$ and DSP motors and can program into a single device $^{84}$.

Intra-internal and high-speed communications allow the full operation to operate on a single device, eliminating the need to transfer data out of the light ${ }^{85}$. Integrated intelligence is one of the areas currently being explored in 5G development. The tools, methods, and techniques we use to build peripherals, cloud software, gateways to connect them, and endusers use different applications for many reasons ${ }^{86}$, e including performance, memory retention, and performance ${ }^{87}$. It means that the duties of different devices are often described as "a priori" during their design and implementation, and we are far from programming software that allows the program to "flow" from one device to another the other (which is called protection) ${ }^{88}$. Without adapting software as part of the future $6 \mathrm{G}$ network, we are still clinging to away we have to decide what will put the intelligence on the network topology during the design because calculations cannot be easily arranged without preparation during design ${ }^{89}$.

\section{Augmented and Virtual Reality}

Because reality and increased reality require less latency and higher bandwidth, all of these applications are very useful from the many registration opportunities. Multi-Access Edge Computing enables fast, periodic data communication between the device and the end of the network ${ }^{90}$. Its proximity provides essential features such as low latency, scalability, and high speed resulting in unparalleled VR / AR experience. The reality of the web is when image computers create a slow-moving world with only ears or glasses to detect $^{91}$ - increasing the reality of computer graphics

\footnotetext{
${ }^{81}$ Condoluci et al., 2018

${ }^{82}$ Chu and Jun 2019

${ }^{83}$ Lin et al., 2016

${ }^{84}$ Sodhro et al., 2018

${ }^{85}$ Mohanta et al., 2019

${ }^{86}$ Trakadas et al., 2019

${ }^{87}$ Vukobratovic et al., 2016

${ }^{88}$ Chen et al., 2016

${ }^{89}$ Hossain and Muhammad., 2017

${ }^{90}$ Zafeiropoulos et al., 2020

${ }^{91}$ Javaid et al., 2018
} 
to create a web world that does not require glass or audio $^{92}$. For example, one might know or play Pokemon Go, which went viral on social media. Pokemon Go is an example of augmented reality because it sees the placement of a computergenerated Pokemon on the voice body's body ${ }^{93}$. Amazon is working with Verizon to bring the cloud closer to mobile and connected devices across the Edge of Verizon's 5G UWB network ${ }^{94}$.

Verizon 5G Edge Computing uses AWS wavelength to allow developers to deliver applications with very low latency to $5 \mathrm{G}$ mobile devices ${ }^{95}$. With this partnership between AWS wavelength and Verizon 5G Edge, developers can offer a wide range of solutions for end-to-end, stand-alone device industry, smart device port solutions. IOTs), AR / VR ${ }^{96}$. To achieve this, the Verizon $5 \mathrm{G}$ Edge enables mobile telephony and seamless communication between users, devices, and applications ${ }^{97}$. AWS wavelength allows customers to use application components with very low latency at the edge of the network and then connect seamlessly to all clouds running in AWS ${ }^{98}$. With Verizon's ultra-wideband 5G and AWS, customers can provide applications and solutions with higher speeds, more security, and ultra-low latency. The study is the development of a new cloud technology called Orion. It is designed to improve the quality of video game streaming ${ }^{99}$.

The combination of Orion, AWS wavelength and the Verizon 5G network helps deliver smooth, ultra-low latency flow, allowing millions of players to play games efficiently at their highest levels. This technology allows gamers to play their favourite games anywhere, anytime now without downloading or purchasing expensive devices ${ }^{100}$. There is a profound impact on the inside and outside of the field. The Verizon 5G Edge enables mobile telephony and seamless communication between users, devices, and apps to achieve this. All of these applications already have cross-computing opportunities and widespread use ${ }^{101}$. However, with

\footnotetext{
${ }^{92}$ Rashid and Abd Razak., 2019

${ }^{93}$ Alsaeedy and Chong., 2019

${ }^{94}$ Velev et al., 2018

${ }^{95}$ Sharma et al., 2018

${ }^{96}$ Adeel et al., 2018

${ }^{97}$ Zikria et al., 2018

${ }^{98}$ Asif et al., 2020

${ }^{99}$ Hayes and Omar., 2019
}

the implementation of 5G, applications should grow and thrive shortly ${ }^{102}$.

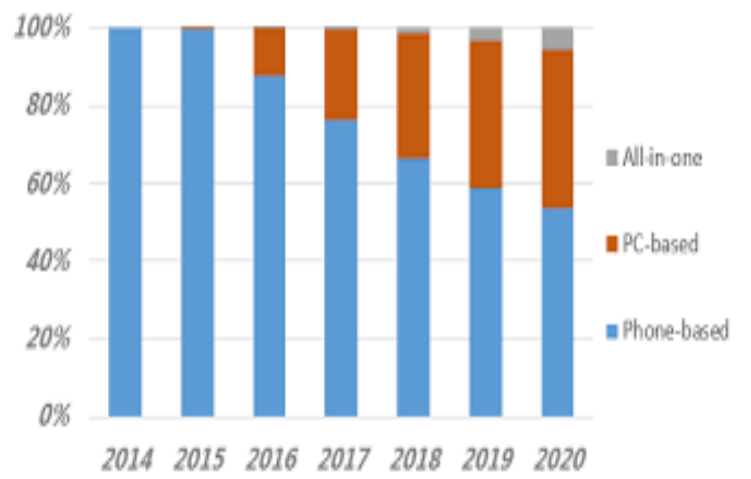

(Source: Hayes and Omar., 2019)

\section{A. Healthcare}

As technology as a whole has evolved, there have been dramatic changes in health. Some devices, such as Fitbit, glucose, check, and other telemedicine equipment, are changing this category. The data stored and processed on these devices used to update patient data ${ }^{103}$. However, the cloud of these developments is unable to process and store the amount of information created for millions of patients. Multi-space computers can be a saviour in this situation by combining these machine tools with the provision of trusted physicians and clinicians, near-patient information, especially in emergencies $^{104}$. Also, the Creative Institute of Technology at the University of Southern California used real-life recreation to improve mental health, contributing to the foundation of intellectual, emotional, and social models. To demonstrate the power of facts in influencing people's emotions, they put people in simulations to stimulate emotions and then teach them to adapt those emotions well ${ }^{105}$.

\section{B. Automated Vehicles}

Not to mention that automated vehicles are efficient enough to perform all types of tasks on a computer and are connected enough to interact with more than one network or device. These automated vehicles are in constant communication around the world and the end, do the same thing in a second phase based on information from a wide range of intelligent sensors ${ }^{106}$. Independent vehicles can transmit information on road conditions and climate changes so that other vehicles are aware of hazards such as accidents, rubbish, flooding and evacuation

\footnotetext{
${ }^{100}$ Sk and Masilamani., 2018

${ }^{101}$ Monge et al., 2018

${ }^{102}$ Ahmed et al., 2019

${ }^{103}$ Nieto et al., 2019

${ }^{104}$ Condoluci et al., 2018

${ }^{105}$ Rezvy et al., 2019

${ }^{106}$ Sodhro et al., 2018
} 
information to deal with the situation. Despite the steady flow of information, there should be free access to the centres to check important information for later analysis ${ }^{107}$.

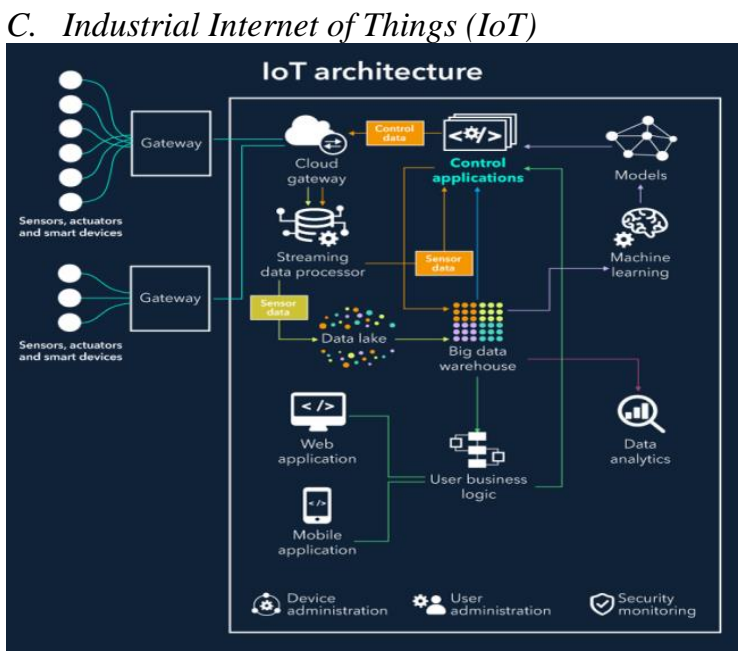

(Source: Lin et al., 2016)

One of the changes associated with the expansion of the Internet of Things has taken place in business which can be called the Internet of Things Engineering or IIOT. However, there is a safety issue which needs to resolve immediately in order to maintain sustainability ${ }^{108}$. Using the software and the tools it provides, we improved security and provide with necessary information about devices, devices, tools, and vehicles to enable employees to work in a safe environment ${ }^{109}$.

\section{G Communication}

IoT peripherals and the development of multi-space computing infrastructure expected to enable the adaptation of existing $5 \mathrm{G}$ networks. About $4 \mathrm{G}$ and how devices come today, the speed, quality and bandwidth of operation are lacking as a long-term solution in a world dominated by stand-alone vehicles ${ }^{110}$. $5 \mathrm{G}$, on the other hand, can provide businesses with ways to increase computer bandwidth and network bandwidth. A large number of $5 \mathrm{G}$ towers is sure to enable the future of the independent vehicles mentioned above $\mathrm{e}^{111}$.

Data Analytics

It estimated that one drive could generate about 30 terabytes of data per day. More than 250 million vehicles in the United States alone, and 10 million are projected to be out of service by the end of this year, so consider how much information there will be $\mathrm{be}^{112}$. All data generated are fragmented and unstructured,

\footnotetext{
${ }^{107}$ Chu and Jun 2019

${ }^{108}$ Lin et al., 2016

${ }^{109}$ Mohanta et al., 2019

${ }^{110}$ Vukobratovic et al., 2016
}

and these databases must be processed with robust data analysis software to generate business-benefit information ${ }^{113}$. Multiple Access Edge Computing can help prioritise which data should be put on edge for further operations and should be returned to the data centre for details. It acts as a relay site and provides additional computing power for mission-critical details that must remain with users ${ }^{114}$.

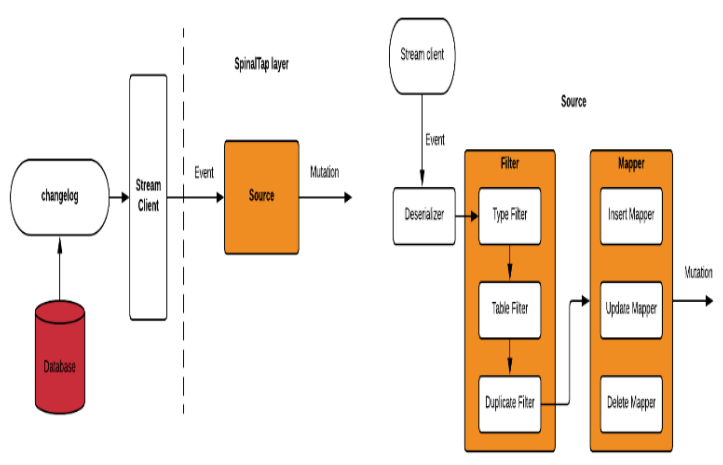

(Source: Zafeiropoulos et al., 2020)

\section{Artificial Intelligence in MEC on $5 G$}

By 2024, 5G Multi-Access Edge Computing (MEC) is projected to be a multi-billion-dollar industry, with large commercial organisations making \$ 73 million. The complexity of the information changes every year. The emergence of unpredictable organisational framework conditions is based on the expansion of flexible administrations. Web access providers must generate traffic to use the Internet, partner vehicles, Internet videos, Internet games, Voice over IP, and Internet of Things (IoT) devices. As mentioned above, the new requirements for on-demand benefits require a sea change compared to organisations with fixed and mobile access. Various 5th century $(5 \mathrm{G})$ organisations were created to cater to the movement's interest and diversity. Network administrators are given distributed computing to handle the unusual traffic demanded by today's customers. 5G organisations will leverage planning capabilities (SDN) and organisational virtualisation (NFV) to reduce mobile organisation development's operational costs and realise benefits on demand. End customers can expect long-term improvements as $5 \mathrm{G}$ has taken steps to provide low inertia, high availability and high bandwidth adaptation in a wide variety of use cases, including lagged applications such as stand-alone vehicles and automated industrial 4.0 robotics.

\section{E. Market Research on MEC}

Considering the need for a new generation of communication, many researchers have made

\footnotetext{
${ }^{111}$ Trakadas et al., 2019

${ }^{112}$ Chen et al., 2016

${ }^{113}$ Zafeiropoulos et al., 2020

${ }^{114}$ Hossain and Muhammad., 2017
} 
every effort to further develop the MEC. The Industrial Telecommunications Division (ISG) of the European Telecommunications Standards Institute (ETSI), introduced the MEC concept, has begun building open environmental standards ${ }^{115}$. Allows service and services to integrate user manuals with ease. Many of the members have set their goals of contributing to SMEs' development, not only members of the communications sector but also manufacturing companies ${ }^{116}$, service companies and universities. Some of these critical keys are Vodafone, IBM, Nokia, Intel and many more. Many issues of the ETSI ISG over the past few years have focused on the general status of the MEC ${ }^{117}$. These publications serve different purposes accordingly. For example, some reports emphasise MEC's everyday use and benefits, while others guide MEC needs ranging from care, operational and safety requirements. ETSI ISG also provided different solutions to many vital issues in the establishment of MEC in different environments ${ }^{118}$.

Although MEC has provided structural and technological support to many applications and services since the inception of $5 \mathrm{G}$, there is a strong need for future research and development to meet the challenges of advancement work ${ }^{119}$.

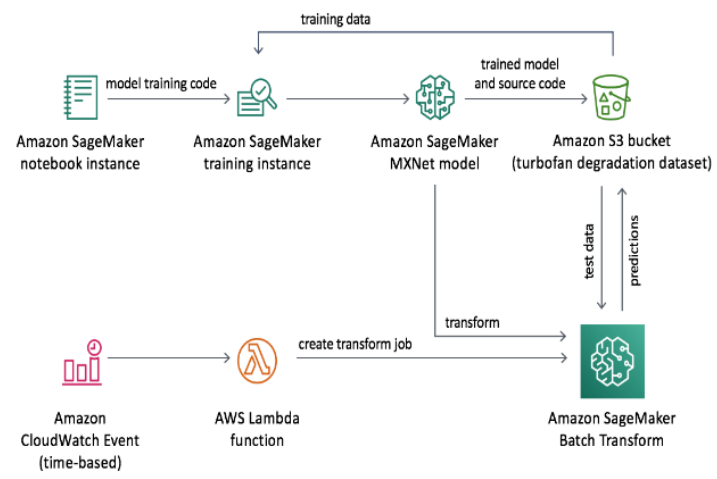

(Source: Javaid et al., 2018)

\section{F. Telecom Industry in a $5 G$ World}

With OTT (ultra-high-end) service companies, telecom operators made a strong impact. OTT messaging services take precedence over messaging, and VoIP has a significant impact on international telephony. Loss of revenue from telecommunications due to OTT services will increase ${ }^{120}$. Now, with $5 \mathrm{G}$, the telecommunications sector needs to make significant changes in the existing business

\footnotetext{
${ }^{115}$ Javaid et al., 2018

${ }^{116}$ Sharma et al., 2018

${ }^{117}$ Rashid and Abd Razak., 2019

${ }^{118}$ Velev et al., 2018

${ }^{119}$ Alsaeedy and Chong., 2019

${ }^{120}$ Zikria et al., 2018
}

environment. These new changes are critical to the telecom operator's continued business. Let us take a look at a few changes to Telecom's business model after $5 \mathrm{G}^{121}$.

\section{1) Connectivity Model}

In this example, the testers run a service. The retail business consumer business is that customers are isolated from the physical network and are not organised. The 5G network has changed this model, allowing operators to use only the parts of the network they need and manage visual data to improve performance and cost savings. It requires a modular structure for $5 \mathrm{G}$ networks ${ }^{122}$.

\section{2) Partnership Model}

This example refers to the relationship of network companies with third-party OTT or virtual network operators. In any case, 5G allows for different interactions between operators and service/application companies ${ }^{123}$. With $5 \mathrm{G}$, one can build a network of analytics components that can provide functionality for all types of network communications. The service can use the policy based on specific ways to regulate it ${ }^{124}$.

\section{3) Digital Service Providers Model}

In addition to the network, network services such as TV and video, financial services, and building experts are also offered. Just by focusing on communication, it will not make any profit in the future of mobile phones ${ }^{125}$.
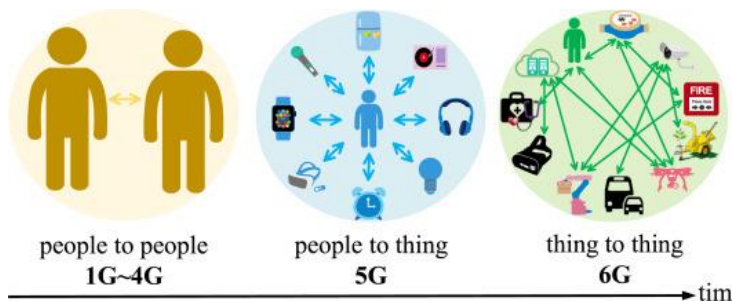

(Source: Monge et al., 2018)

\section{Vision for the 2030s Edge-Driven Artificial Intelligence}

There is virtually no industry that will not affect modern wisdom. It is especially true in recent years, as data collection and analysis are critical due to the reliability of Internet communication issues, the increasing number of network connections and faster computer operations ${ }^{126}$. Regardless of how artificial life affects the lives today, it is difficult to ignore the fact that in the future there will be new and innovative

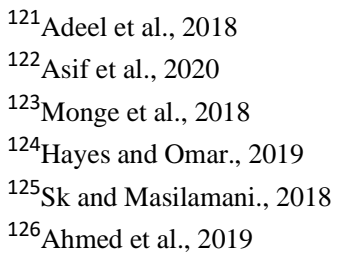


services: (i) transportation and travel in areas that third, (ii) production and industry, (iii) health and well-being, (iv) education and training, (v) media and entertainment, (vi) trade and commerce, (vii) protection of the environment, (viii) service providers ${ }^{127}$. The complexity of implementing the requirements requires increasing expertise in all areas to provide efficient, safe, reliable, and flexible services. Additionally experience from cloud service, we continually facilitate progress from the Internet of Things ${ }^{128}$.

Due to the above requirements, the need for "Internet of Things Smartphone " is also beginning to emerge to make that Internet more reliable, efficient, flexible and secure ${ }^{129}$. Now, 6G connectivity to edge-driven intelligent processors can play an important role. Compared to the advanced computing of cloud services such as Google, Amazon, and Microsoft, it allows for greater integration of computers and networks over $6 \mathrm{G}^{130}$. For example, $6 \mathrm{G}$ base stations can be a combination of intelligent applications that require both classification and linking of resources ${ }^{131}$. It is likely to provide a new opportunity for motorists and, in some cases, forts to restore their central position in the market and increase the additional cost they offer ${ }^{132}$. As smart devices connect in the $6 \mathrm{G}$ era, it is hard to believe that the we will be able to deal with them, the difficulty of their use and working conditions while continuing to use the network in a static, simple and stupid state road ${ }^{133}$.

The same need may arise for any other service using an integrated network input, including telephones, video telephones ${ }^{134}$. Video conferencing, and videoon-demand, streaming and real-time streaming, where wireless communication no longer only provides a "connection" between two or more people or "video channels" at the request of the TV user ${ }^{135}$. However, it requires appropriately certifying all aspects to ensure road safety records, using the chainlink block and identifying unusual or unusual behaviours in real-time ${ }^{136}$. The exchange of data in the model is more than just the exchange of data; it changes the frequency of past, present and possibly the future appearance of these data ${ }^{137}$. In the future, 6G wireless communication, reliability, service

\footnotetext{
${ }^{127}$ Nieto et al., 2019

${ }^{128}$ Condoluci et al., 2018

${ }^{129}$ Rezvy et al., 2019

${ }^{130} \mathrm{Chu}$ and Jun 2019

${ }^{131}$ Mohanta et al., 2019

${ }^{132}$ Sodhro et al., 2018

${ }^{133}$ Lin et al., 2016

${ }^{134}$ Mohanta et al., 2019

${ }^{135}$ Trakadas et al., 2019

${ }^{136}$ Vukobratovic et al., 2016

${ }^{137}$ Chen et al., 2016
}

status, health monitoring, error detection, reliability and flexibility will be the norm, and progressive, smart solutions will be highly promising candidates will make a significant contribution in satisfying these needs ${ }^{138}$.

One can easily predict the fact that much information will be sent to future $6 \mathrm{G}$ wireless nodes, and more and more important-use programs and services will rely heavily on this information ${ }^{139}$. The highly intelligent design will undoubtedly be a useful topic in the future flow of $6 \mathrm{G}$ wireless communications, and at the same time can be a technology that offers a wide range of value-added applications and services ${ }^{140}$. Intelligent information at wireless nodes can provide many advanced QoS services and features for application planning ${ }^{141}$. Current customisation methods used in the cloud cannot be applied directly to the computer due to the variety of primary classifications and distribution of resource information. Because even the solutions available for translating different clouds into a homogeneous platform are not working well at the moment ${ }^{142}$, Mohan explores the challenge of computing (i) limiting equipment, (ii) limiting the environment, (iii) obtaining and reliable, (iv) limiting power) Moreover, offering solutions, which are needed to test the current planning of the cloud environment ${ }^{143}$.

We aim for performance, cost, safety, efficiency and reliability to be the most critical features and measurement methods of any end and end-to-end solution $^{144}$. divide edge intelligence into six levels concerning the amount and duration of download information $^{145}$. The study extends of ${ }^{146}$ the version from borderline DNNs to general AI models and models with seven components where the boundary can be seen as a cluster or cluster of independent, intelligent nodes or a cluster of co-located / integrated boundary nodes ${ }^{147}$. we also add variations in degrees of independence in the activity of peripheral nodes ${ }^{148}$.

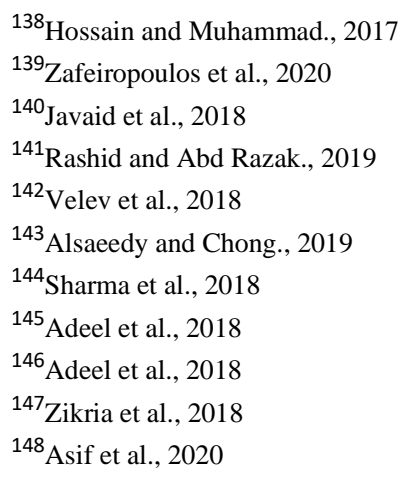




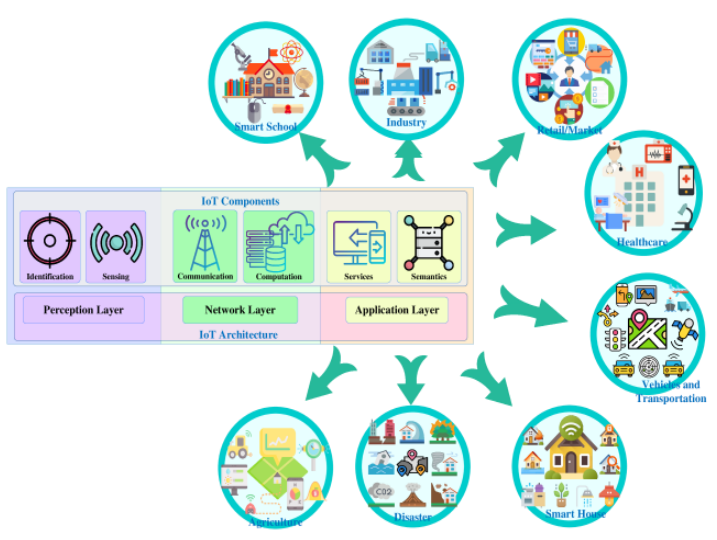

(Source: Adeel et al., 2018)

These aspects of borderline intelligence are described in detail as follows:

- Cloud Intelligence: Learn and see a full-fledged cloud intelligence platform around the world.

- Level 0: Collaborate with the cloud learning platform: Train the cloud computing model in the cloud, and develop the advanced cloud computing environment in collaboration with the cloud endpoint. Now, communication with the cloud means that the data is a decompressed component within the cloud.

- Level 1: In-Edge Co-decision and Cloud Learning: Test ones AI image during the day while running the AI test at the edge. This edge means that the model implemented at the end of the website, which can be accessed by downloading all or part of the data to the bottom or border devices independently or in a system combine $^{149}$.

- Level 2: Information from the device and training in the cloud: Test the intelligence model during the day, but the reason for the intelligence test ordered locally. This device means that no data will be downloaded.

- Level 3: Collaborative training and day-to-day reflection: Training and reflection on the model of intelligence, such as the end-of-day togetherness

- $\quad$ Step 4: Everything on the End: Examples and Ideas of the Wisdom Model on the End ${ }^{150}$.

- Level 5: Border Interoperability Training and Reasoning: Artificial Intelligence Model Training and Reasoning in Border Interaction Mode

\footnotetext{
${ }^{149}$ Hayes and Omar., 2019

${ }^{150}$ Monge et al., 2018

${ }^{151}$ Sk and Masilamani., 2018

${ }^{152}$ Rezvy et al., 2019

${ }^{153}$ Ahmed et al., 2019

${ }^{154}$ Chu and Jun 2019

${ }^{155}$ Nieto et al., 2019
}

- Level 6: All on the machine: Try and describe the specific design made on the machine ${ }^{151}$.

AI Edge and AI Edge can be divided into frames, for example, the edge looks like an "emergency cloud" of network communication, and the "edge node-set" can interact to share information about a specific situation and environment, and computational changes and communication loads ${ }^{152}$. Both during training and during retirement ${ }^{153}$. We then list and summarise critical areas that we believe will be very useful in the future application in any case situation 4. It emphasises where the research is conducted and where the applications located and serve ${ }^{154}$, depending on specific manual conditions, environmental conditions, communication networks, collaborative structures should be disciplined, and practices and costs should be balanced ${ }^{155}$. Some examples of state-of-the-art technology for improving network communications over the $6 \mathrm{G}$ era and for control over life (AI for Edge). Edge is a platform for distributed application-focused smart services (AI of Edge) ${ }^{156}$.

There is no cost because it is also essential to reduce the reliance between $\mathrm{AI}$ and Edge for $\mathrm{AI}$ to implement efficient, secure, reliable and flexible services over $6 \mathrm{G}^{157}$. Development and institutional frameworks should ensure consistency and quality together ${ }^{158}$, but may not necessarily require full implementation of these services at all times. For optimal adaptation, we may need to develop a "6G network ontology" to build all appropriate combinations of "micro-services" at the end nodes ${ }^{159}$. The benefits of enlightenment are enormous, but the application of knowledge (learning) in addition to the application of reasoning (reasoning) poses many technological challenges, unlike traditional practices, of intelligence ${ }^{160}$. Therefore, it is necessary to identify and analyse these end-of-life problems and look for new hardware and technology tools. From this point of view, many serious problems associated with the edge of intelligence and some critical solutions are discussed below ${ }^{161}$.

\section{G. Edge Infrastructure Solutions}

The top of the network interface is the best example of the MEC map currently being compared by ETSI. The architect describes the framework's components, roles and responsibilities, system APIs, and interactions for collaboration and third-party system integration $^{162}$. The expectation is a wide-open

\footnotetext{
${ }^{156}$ Condoluci et al., 2018

${ }^{157}$ Vukobratovic et al., 2016

${ }^{158}$ Sodhro et al., 2018

${ }^{159}$ Lin et al., 2016

${ }^{160}$ Mohanta et al., 2019

${ }^{161}$ Trakadas et al., 2019

${ }^{162}$ Chen et al., 2016
} 
space from a wide range of customers, so it guides on implementing processes and policies based on the artist's reference and validating its ideas ${ }^{163}$. While the implementation of data for communication and interaction areas is still open, the foundation based on integrated processes and management in two dimensions: system performance and hosting status ${ }^{164}$. However, the central component expected to have independent power over all applications. Establish and host status groups operating under the direction of the semi-autonomous orchestra to direct their resources to their group ${ }^{165}$. These components expected to be provided by the signal processor in their operation.

These policies are unlikely to work, but they do create opportunities to re-engage in activities that take less time to use multiple lists, use the information and compile, share program information, etc. in rugged IoT environments ${ }^{166}$. $n$ terms of competent capabilities in peripheral infrastructure, broad-based deployment and time-sharing data sharing processes, operations systems, key indicators $(\mathrm{KPI})^{167}$. The data submitted, QoS, and interaction quality (QoE), and more building, training, and make different predictions to better behaviour is mostly unwritten. For example, different approaches are available $^{168}$. ETSI MEC is a two-tier modelling and management system in the cloud or on the platform and similar to the web components ${ }^{169}$. Fog computations, on the other hand, provide a hierarchical computation platform for the whole operation, usually limited by the state in which the computational unit is located, i.e., the fog nodes, which are still in the right positions. in the cloud ${ }^{170}$.

Cloud computing is a similar concept when it is possible to use the power of the web on the web on the supply on demand and under / overuse the power to distribute based on cost an extension of the administration of benefits ${ }^{171}$. In a small environment, the role of independent and local authorities increases in importance and complexity. Foggy computing has recently come to the fore, with data-driven IoT devices, such as WSNs and mobile devices, already being used for data mining for some applications in the data source ${ }^{172}$. Another approach to classifying a cloud computing device's status, which allows the EU

\footnotetext{
${ }^{163}$ Hossain and Muhammad., 2017

${ }^{164}$ Zafeiropoulos et al., 2020

${ }^{165}$ Rashid and Abd Razak., 2019

${ }^{166}$ Velev et al., 2018

${ }^{167}$ Alsaeedy and Chong., 2019

${ }^{168}$ Sharma et al., 2018

${ }^{169}$ Sharma et al., 2018

${ }^{170}$ Adeel et al., 2018

${ }^{171}$ Zikria et al., 2018

${ }^{172}$ Asif et al., 2020
}

to integrate allocations for the end-to-end cloud ${ }^{173}$. In addition to the MEC model, there are open source solution solutions for top-level management, such as Google Kubernetes and Docker Swarm that widely used in industry ${ }^{174}$.

\section{H. Edge Platform Orchestration}

The open nature of the CFD environment and the general body of the boundaries of computer systems justify the use of intelligent technology to streamline and regulate such systems ${ }^{175}$. Also, implementing essential promises such as high efficiency and QoE requires a wide range of complex information and data processing algorithms ${ }^{176}$. These types of structured algorithms will be difficult to initially design and develop and then take, maintain and evaluate ${ }^{177}$. BIn addition, the distributed running an end-to-end segmentation fits in well with the building foundation. The overall challenge is to solve the distribution of problems, which means that the important thing is that there are physical conditions and what features needed in every appropriate place to support on the body network topology ${ }^{178}$. A popular way of providing the element with an existing asset, such as wireless base stations, in terms of having essential network connections, or using low-resource, location- basic computational modules, such as cloud computing ${ }^{179}$. These uses are limited by budgets and public disclosures and should be carefully planned first, for example, based on historical data and in response to activities and visual cues $^{180}$.

\section{Data and Network Management}

Obtaining and archiving types is essential at the mine end. Unlike the integration of collective thinking, in many cases, the idea of using knowledge is based on "small data"181. Therefore, the general trust of the expert in visual information is an important task. On the other hand, although peripherals contain a large amount of information, it is essential to identify sequential and illegal links order to complete the expansion of information to avoid malfunctions (due to over-installation, for an example) in intelligent models ${ }^{182}$. sIn addition, dependencies rely on the light source and can produce different types of information and multisensory (audio, video, touch screen), spatial, temporal, and stochastic

\footnotetext{
${ }^{173}$ Hayes and Omar., 2019

${ }^{174}$ Monge et al., 2018

${ }^{175}$ Sk and Masilamani., 2018

${ }^{176}$ Ahmed et al., 2019

${ }^{177}$ Nieto et al., 2019

${ }^{178}$ Condoluci et al., 2018

${ }^{179}$ Chu and Jun 2019

${ }^{180}$ Rezvy et al., 2019

${ }^{181}$ Sodhro et al., 2018

${ }^{182}$ Lin et al., 2016
} 
properties $^{183}$. Also, since these heterogeneous sensory data are integrated into a wide range of networks, the same data are likely to contradict each other $^{184}$. Thus, the combination of these different types of data affects the performance of the light end $^{185}$. In addition to information, communication levels and needs can change over time, even for short periods (work-critical requests) requiring short response times ${ }^{186}$.

In the face of such changes, such models may not be able to adapt or adapt to different ways. The distribution of data pieces and the experience of experimental intelligence rather than raw data significantly reduces the overall volume of any network connection and increases the potential number of software applications that can be executed in a short period ${ }^{187}$. Therefore, it is necessary to clearly understand and explain the information, origin, and life cycle of the model and provide measurements to compare the models with their value and current status ${ }^{188}$. Information preparation for machine learning is essential for the effective and robust construction of scientific models in general, cloud, and data centres ${ }^{189}$. It is possible to use sophisticated methods to classify similarities and use tools to find differences in information ${ }^{190}$. After classifying the above, subsampling methods can be used to regulate the smart boundary community's production. To solve problems of a large proportion of data, peripherals need to model time at the cost of power to obtain large amounts of information ${ }^{191}$.

Also, synthetic information can be obtained by referring to well-learned general competition networks ${ }^{192}$. Also, the use of additional learning methods to teach higher levels over time and the introduction of well-learned methods by gaining knowledge and ways to connect the ways of good problems in low-income cases ${ }^{193}$. Border control, which must deal with the integration of different types of data, can be used to extract samples (e.g. training samples) and share training in multiple ways $^{194}$. To eliminate the conflict in different emotional data, background information can be used in social media communication networks to

\footnotetext{
${ }^{183}$ Mohanta et al., 2019

${ }^{184}$ Trakadas et al., 2019

${ }^{185}$ Chen et al., 2016

${ }^{186}$ Vukobratovic et al., 2016

${ }^{187}$ Hossain and Muhammad., 2017

${ }^{188}$ Zafeiropoulos et al., 2020

${ }^{189}$ Javaid et al., 2018

${ }^{190}$ Rashid and Abd Razak., 2019

${ }^{191}$ Velev et al., 2018

${ }^{192}$ Alsaeedy and Chong., 2019

${ }^{193}$ Sharma et al., 2018

${ }^{194}$ Adeel et al., 2018
}

reconstruct and restore permanent data ${ }^{195}$. Edge AI can be used to improve the operation and performance of network nodes ${ }^{196}$. However, in some cases, such as errors in retrieval and recovery, the problem of errors is rarely found, and therefore the use of machine learning is very unbalanced ${ }^{197}$. In general, communication and events are often the same, meaning that, for example, means of communication are ways to identify ways in which effort can be met with massive effort ${ }^{198}$.

If machine users and startup users start using greedy AI flow controls and route / GW options, network traffic can be much more difficult to predict ${ }^{199}$. Although many methods can be used to generate data production, it is not always clear that such information is best for training ${ }^{200}$. For example, dance track search. One option is to use modelling to obtain appropriate data balances, which require some kind of network number twin point. Also, as portable radio transmissions move toward viewing milestones (and more) 201 $^{2}$ it may be useful to integrate other types of information into communications management, such as detailed information on weather conditions (heavy rainfall) and seasonal changes. In summary, it is better to understand the behaviour of a website than to treat a black box ${ }^{202}$.

\section{$J$. The Intersection of Edge and Device Intelligence} The end-of-life application location is critical for time-response and response in the environmental environment and operational activities ${ }^{203}$. Since then, there have been road-driven methods that include mobile devices as part of computing platforms, including mobile services, Mobile Edge and Mobile Fog, to cross the border of distributed distribution. Typically ${ }^{204}$, these low-level conditions have potential limitations for the operation, analysis, and distribution of "small data," which advanced detail requires additional support from the side ${ }^{205}$. Therefore, such distributed applications are often distributed among users of computing and peripheral systems and cloud computing ${ }^{206}$. In this case, the sharing of (ongoing) national data and assets, both cross-platform and top-down, is essential to save

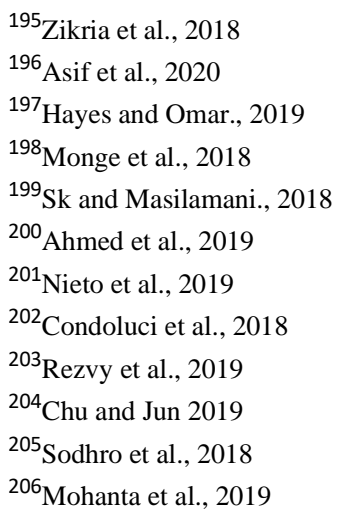


tools, such as power, access to devices, and prepared competent response strategies to users ${ }^{207}$. Topical issues of collaboration, such as uniform uniformity for data sharing, results, actions and high-level sequential competencies (e.g. algorithms) ${ }^{208}$. Also, the large scale of a combination of web-based integration dramatically increases the scope of management and classification for a broader view of program performance. It promotes the limitation of content-restraint ${ }^{209}$.

Therefore, pure ring-shaped solutions are already required in the mobile to increase independence and original features. Thus, the traditional model of distributed AI, software development, and multiagency systems has been well demonstrated in the delivery of independence ${ }^{210}$, operations, adaptation, machine learning, number portability and collaboration capacity, and even limited IoT devices ${ }^{211}$. Such devices are often called Things Wise in the case of Internet Things. Current use of day-today connectivity devices includes object modelling, configuration and direct collaboration, shared management and management (e.g., SDN), and system, communications, and monitoring devices ${ }^{212}$. The question of how to expand competency from reemployment to motivating factors that have competent skills such as learning and action is still open. Other intelligent methods that facilitate the integration and interoperability of collaborations and interactions include multidisciplinary, sports games, and genealogy algorithms ${ }^{213}$.

\section{K. Software Development for Edge}

Edge software development is based on virtualisation, such as virtual machines and lightweight containers. The back-end books are developed as a programmatic program that can be implemented by multiple partners ${ }^{214}$, which automatically builds user-generated images and administrative groups that support the vitality of the applications ${ }^{215}$. Images are provided to peripheral hosts on virtualisation infrastructure by system policies, then managed and built with standards and hosting components that are additionally responsible for providing software-specific services ${ }^{216}$, information and network communication, and

\footnotetext{
${ }^{207}$ Lin et al., 2016

${ }^{208}$ Vukobratovic et al., 2016

${ }^{209}$ Trakadas et al., 2019

${ }^{210}$ Javaid et al., 2018

${ }^{211}$ Chen et al., 2016

${ }^{212}$ Hossain and Muhammad., 2017

${ }^{213}$ Zafeiropoulos et al., 2020

${ }^{214}$ Rashid and Abd Razak., 2019

${ }^{215}$ Velev et al., 2018

${ }^{216}$ Alsaeedy and Chong., 2019

${ }^{217}$ Sharma et al., 2018
}

maintenance required $\mathrm{QoE}^{217}$. The challenge, in addition to the implementation of ideas, is to lead policies for service standards ${ }^{218}$, rights, billing, and other software packages and to coordinate and improve their use online, maybe with other groups service and communications companies ${ }^{219}$. When one set up and operate critical applications, compress and pull methods are simplified by not opening the operation and transferring user-friendly objects to a nearby location ${ }^{220}$.

Also, from the standpoint of independence, limiting the serial number of software components is a supporting tool. In this regard, security and sharing of resources and information are important issues ${ }^{221}$. Standard machines typically contain monolithic discrete application applications, typically large gigabytes, which provide resources to supply and travel around the environment ${ }^{222}$. A distributed approach to application development is a microservice designed to separate modular development application components in the form of self-contained applications that can be used to create an application workflow ${ }^{223}$, or and required. Now, it is a clean version of the web-based, container, microservice storage for use ${ }^{224}$, which can be operated as horizontal or non-horizontal devices, are a substitute for containers. The number of images of a nice reelbased microservice can be very similar to other containers, and therefore the core uniqueness can provide adequate separation and similar resources ${ }^{225}$. DevOps policies, such as CI / CD, provide tools for microservice development exceptions, version control, and automated delivery of such components using administrative components ${ }^{226}$.

Managing a large web site is, of course, challenging, as small add-on nodes and their channels greatly enhance the management and implementation of monitoring systems and packages ${ }^{227}$. Many AI-based CI / CD-based technologies, such as Kubeflow9 and MLFlow10, support channels for the development, learning, and delivery of cloud-based digital information $^{228}$. However, the essentials serve as environmental sciences; however, such structures

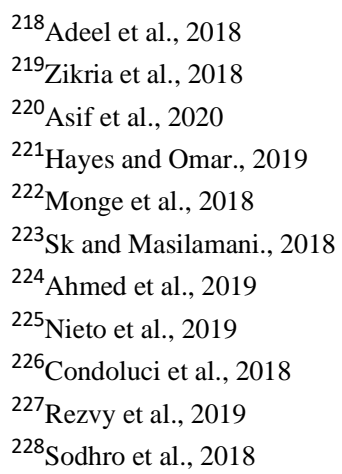


should be adapted to the information endpoint and connected to the cloud environment ${ }^{229}$.

\section{A USE CASE TOWARDS EDGE INTELLIGENCE IN IOT}

In this section, the we recommend using a case using MEC in IoT environments, such as proximity to intelligent travel technologies, which can help to understand the wisdom of the IoT.

The immediate problem is to find the buyer everywhere nearby. In the case of ITS, tens of thousands of passengers (or to speak, customers) continue to travel on the road, their proximity needs regular check-ups, in order to save safe roads ITS. A close-knit problem requires time-consuming solutions and low costs. For this reason, we recommend the MEC-enhancing intelligence available close to the solution to reduce latency and currently recognising near-earning revenue.

\section{MEC-updated drawers for close entry}

Our MEC-enhanced image resolution is shown in Figure 7, which fully utilisesMEC features, such as real-time data, high-bandwidth, and low latency. In this architectural design, individuals consult with their new MEC service, rather than accessing the website to contact the central service, to ensure a low level of architectural quality. While both the MEC server and the central server are affected in Figure 7, note that the MEC service makes it easier to reach more customers, but the 'central office' is only affected when arrived from that small number of depositors on top. For ease of reference, we use the term "friend name" to refer to people living close to the patient. Apparently, for most people, all of their partners are in the same MEC service. However, some establishments, and a small number of establishments on the MEC border service, maybe at the service of one MEC employee, and others may be available at the services within a group of another MEC employee, as well as communication with another MEC employee. In this case, the central service is used to implement the closeness of these minorities, after receiving the information of these heroes as previously reported by MEC staff. In such cases, the patient exchanges regular information. MEC supply

\section{B. Quick access algorithms}

Another reason for the proximity is the reduced cost of communication resulting from the exchange of information between people and services. To achieve this goal, we designed a "secure" mobile region for individuals. As long as the client is in a "secure" mobile region, there is no need to update his / her data on the supply, thus reducing the cost of communication. It seems that regional mobile security is associated with the importance of regional security circuits. After thousands of experiments, we found that more cycles result in better mobile security protections reaching moderate costs. To obtain this optimal cycle, the we devised a deep-multivariate parameter regression method to obtain the latency, which automatically classifies the best cycles of the same mobile protection region with different limitations integrated into the communication path, such as proximity to the door, the speed of the public, the average density of customers, and the total number of mobile phone users.

We use five types of neural networks, including neural networks, neural networks (CNN), neural networks (RNNs), short memory (LSTM) neural networks, and gated recurrent unit (GRU) neural networks. Samples are made on three-way routes, i.e., Oldenburg1 road network, San Joaquin road network2, and the New York City (NY) area network3, pNY for short. And, the major roads are defined by $[0,1000]$ by $[0,1000]$. Moreover, the road network can be divided into four equally-large aids, allowing us to provide four MEC services. Suppose $\mathrm{L}$ means the length of the side of the road square, then each MEC service has a large area with $r=2 L$ of its circumference.

\section{1) Experimental Results of the impacts of MEC on} Latency:

The long side of the Oldenburg, San Joaquin, and New York highways, i.e., L, is more than $100 \mathrm{~km}$. Ideally, place $\mathrm{L}$ at $100 \mathrm{~km}$. Moreover, the distance between $\sqrt{ }$ central service providers can reach 1002 , i.e., $141 \mathrm{~km}$, if we use the combined endurance combination instead of MEC improved structure. In comparison, in planning MEC home improvement, the distance from the patient to him when the we use the four MEC 'final services in the model of road conditions. Yes, more (e.g., 9, 16) MEC services can be provided to service the road network if the road network is divided into more (e.g., 9, 16) organisations, and therefore more or less, e.g., $r=100$ $\sqrt{2} \approx 23.6 \mathrm{~km}, \mathrm{r}=100 \sqrt{ } 682 \approx 17.7 \mathrm{~km}$.

In the model, we compare integration after providing three MEC services with one day-to-day service of $35 \mathrm{~km}, 23.6 \mathrm{~km}, 17.7 \mathrm{~km}$, and $141 \mathrm{~km}$ from the building. Figure 9 shows the RTT latency results. It is clear that the MEC application latency is one-sixth of the service distance, i.e., less than $1 \mathrm{~ms}$, Therefore, it can be concluded that the build can significantly reduce the latency network the MEC, which satisfies low-latency conditions.

2) Example Results in Deep Learning Algorithms: The result of the actions of the five AI algorithms in terms of the loss and accuracy of the numerical value of the epochs are shown in Figure 10, in which the loss includes loss of training data and loss of data evidence are shown at the bottom right of Figs. 10 (a), 10 (b), 10 (c), 10 (d), and 10 (e) but the exact value

${ }^{229}$ Chu and Jun 2019 
of the period is shown below the categories of all the letters. We list the limitations of the five models to be combined in Table III. From Figure 10, we can observe that the LSTM and GRU neural networks have the highest prognosis $(78.84 \%$ and $79.19 \%)$, and do their best to solve this problem., which demonstrates the usefulness of MEC-enhanced nearacquisition intelligence.

\section{1. $5 \mathrm{G}$ AND IOT: CHALlENGES AND OPEN ISSUES}

Border control brings wisdom to the edge. This refers to the ability to search local data, where data is stored, rather than sending it to the cloud for details. Although methods methodsmethods used in communications $^{230}$, scholar, a crucial part in implementing billion VGDU mobile communication device experience the world, not studying ${ }^{231}$. Edge intelligence includes intelligent communications from terminals to end-services, intelligent communication between end-services, details of local behavioural services at the end, local data analytics at the end, not the cloud, to operate a world of trillions of IoT devices and learning devices ${ }^{232}$. Within a network, boundary performs a touch ones internet connection does not exist, and there are no-cost problems. There are currently borderless jobs available: Many stories have already been explored on borderline intelligence. The authors use machine learning in a low-speed FiWi (Fiber-Wireless) network and strive for a more seamless, deep communication experience $^{233}$.

They use multilayer perceptron c (MLP), a type of parameterised neural network (ANN) model, on the basis that they provide an essential module of the perceptual model (EBP) to detect missing responses to traits follow ${ }^{234}$. Authors use intelligence to facilitate the processing of critical, user-friendly applications from the core network to peripheral networks to improve IoT accuracy and responsiveness $^{235}$. They help developers develop their local skills. They can build intelligent services within their WuKong intraocular centre- centric middleware to promote intelligent IoT services by expanding the prototype ${ }^{236}$. The authors explored the need to bring AI edge to IoT devices, so IoT devices

\footnotetext{
${ }^{230}$ Lin et al., 2016

${ }^{231}$ Mohanta et al., 2019

${ }^{232}$ Trakadas et al., 2019

${ }^{233}$ Vukobratovic et al., 2016

${ }^{234}$ Chen et al., 2016

${ }^{235}$ Hossain and Muhammad., 2017

${ }^{236}$ Mohammadkarimi et al., 2020

237 Javaid et al., 2018

${ }^{238}$ Rashid and Abd Razak., 2019

${ }^{239}$ Velev et al., 2018
}

and expanding intelligence can detect, create, and operate. Thus, the use of intelligence is available for many new publications ${ }^{237}$. The authors will develop a comprehensive set of policies, taking into account limited traffic and travel time. They provide a timely and professional schedule to meet these challenges ${ }^{238}$.

Developing a solution for the on-premises, and useful resources of IoT devices, authors use the information on the network interface (NDN) ${ }^{239}$, to accurately estimate the cost of the download. KDN contains a new model of intelligence, S DN, and data analysis (NA). Problems of Border Intelligence: Although many methods are designed to solve the problem of multitasking, most of the time, it remains necessary to have no studies ${ }^{240}$. They are integrating workstations with MEC servers or other environments to save power/cost or achieve faster planning. Different factors can affect stream classification $^{241}$, such as bandwidth access, cloud appearance and availability ${ }^{242}$. EU (user tools) quality, radio and quality control, cost power for local use of applications, or extensive data to download and customise applications ${ }^{243}$. Therefore, to achieve a smart load, many limitations must be considered, as it is a very complicated process and is still a significant problem ${ }^{244}$.

Even though it is rapidly changing technological advances of knowledge, such as in-depth learning and empowerment of the mind ${ }^{245}$, the boundaries of research through the study of large amounts of historical information and positive responses to a force Demonstrate how to develop a knowledge base for effective travel choices ${ }^{246}$. Service remains a significant concern. To make better use of technical knowledge to make decisions on translation services $^{247}$. The importance of challenges must be addressed the choice of source information; then we require the elimination of data that does not solve the problem of large amounts of data ${ }^{248}$. The design of a smart system based on an example of algorithms to choose the most integrated MEC internally. Another challenge needs to be addressed for the technology to reach new technological development ${ }^{249}$. For example, computer instability, computer speed, heterogeneous integration using the system-in-pack

\footnotetext{
${ }^{240}$ Geller and Nair., 2018

${ }^{241}$ Sharma et al., 2018

${ }^{242}$ Sharma et al., 2018

${ }^{243}$ Zikria et al., 2018

${ }^{244}$ Adeel et al., 2018

${ }^{245}$ Zikria et al., 2018

${ }^{246}$ Asif et al., 2020

${ }^{247}$ Vilalta et al., 2017

${ }^{248}$ Monge et al., 2018

${ }^{249}$ Sk and Masilamani., 2018
} 
method $(\mathrm{SeP})^{250}$, and a new class of low-cost. It is an electric motor, and it consumes not only the answers to all the solutions that need to be developed. Green MEC Compared to the Cloud Server Center, each MEC service consumes less power ${ }^{251}$.

However, many MEC installed services still consume much power. Thus, green MEC software's design becomes a critical one ${ }^{252}$. As mentioned, MEC greens are more challenging to design than contact lenses. The reasons are: the possible use methods contact the atmosphere in the green MECs ${ }^{253}$, the first article of green network Center network (DCNs) does not apply to green MEC because they do not recognise radio regulatory management, and the highly unpredictable load model $^{254}$, poses significant challenges in the management of MEC tools. How to implement Green MEC: There are several ways we have suggested achieving the Green MEC goal. For example, three methods are shown in the design of green MEC system ${ }^{255}$, namely, renewable energy MEC system, geographic load balancing (GLB) for MEC equipment, and quantity. Broad parameters for the strength of the MEC system ${ }^{256}$. MEC systems used by energy-efficient to expand Advanced Energy Harvesting Methods (EH) methods allow renewable energy sources (e.g., wind power) to be used as a suitable type of power supply, which is motivated by renewable energy, children from the MEC's ability to obtain $\mathrm{it}^{257}$

Work-in-progress, such as MEC's renewable energy research solutions, focuses on MEC-based EHservers and, similarly, a mobile-based EH-service ${ }^{258}$. However, these two articles, merely discussing the MEC, do not provide appropriate answers for much of the MEC. Geographic Load Balancing (GLB) for MEC an essential technology for green DCNs, GLB can also be performed in MEC environments ${ }^{259}$. For example, thanks to MEC servers and the GLBcollaboration heavy-duty methods, the MEC Server can provide work at near-end workloads at a low $\operatorname{load}^{260}$. Not only does this help a low-end Edge server to boost user power and experience, but it can also extend the life of the mobile device, as it can save performance the server nearest to ${ }^{261}$. Note that GLB

\footnotetext{
${ }^{250}$ Nieto et al., 2019

${ }^{251}$ Ahmed et al., 2019

${ }^{252}$ Sheikh et al., 2018

${ }^{253}$ Dai et al., 2019

${ }^{254}$ Chu and Jun 2019

${ }^{255}$ Lujá et al., 2019

${ }^{256}$ Mohanta et al., 2019

${ }^{257}$ Lin et al., 2016

${ }^{258}$ Trakadas et al., 2019

${ }^{259}$ Soatti et al., 2019

${ }^{260}$ Stanley et al., 2018

${ }^{261}$ Zafeiropoulos et al., 2020
}

implementation may require top-notch service management resources ${ }^{262}$.

Dynamic MEC increases compared to power consumption: MEC labour consumes about $70 \%$ of its energy, even when not slowing down when running at full speed, resulting in power-rated supplies $^{263}$. A composite shared power is a service whose power is used compared to its load configuration. The slow or shut down the speed of some services at the bottom is, for example, waxes referred to texts such as the rehabilitation of the capabilities of services ${ }^{264}$. MEC Repeat: Although the researchers recommended the above methods ${ }^{265}$. There are also problems with green MEC. First, renewable energy MEC systems, strong green decommissioning readings and shared resources are significant challenges that need to be addressed ${ }^{266}$. For large-scale models, the first MEC GLB solutioncentric over clean environment becomes more difficult in MEC environments due to the large differences between DCN and MEC systems ${ }^{267}$.

Second, many critical factors need to be considered when applying GLB technology to the MEC system. One should keep an eye on the superb connectivity when one starts the GLB because the network down to pass when moving work ${ }^{268}$. This results in a large amount of electricity consumption for the machine tool, which allows it to use another service for travelling work. There is a trade-off between latency reduction and regenerative energy, so when using the GLB, service relationships with MEC operations should be considered ${ }^{269}$. Web services can also provide important functions in remote areas of cloud service due to daytime practices, which are difficult to ascertain ${ }^{270}$. Third, efficient sizing is a quick way to close service ends because it does not always work. Indeed, it is necessary to look at the MEC machine loaders' design to strongly select the correct $\operatorname{accuracy}^{271}$.

Unlike conventional DCNs, it can simplify the implementation of sample histories, and predict job modelling for leaders of challenging areas ${ }^{272}$. With a small footprint, the load model on Edge services can

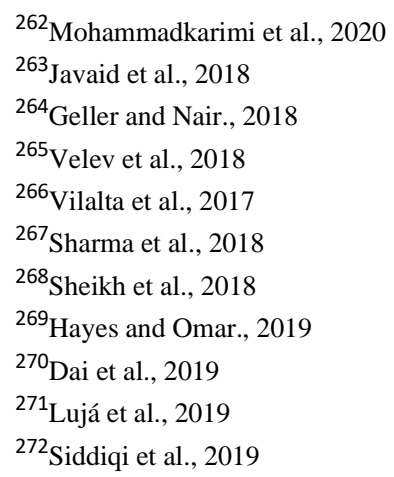


be affected by many factors, such as mobility, temperature, and the number of Edge services across borders, weather, and time and place of service ${ }^{273}$. Therefore, more sophisticated methods are needed to better classify and progress to accelerating and rapidly changing workloads ${ }^{274}$. Also, researchers and developers need to develop the best web algorithms to determine exact quantities ${ }^{275}$, which will require less data. in the future. C. Installation from MEC services and systems) MEC Installation Articles: MEC uses the featured text ${ }^{276}$. In a simple MEC deployment scenario, MEC operates as a separate sandbox standpoint that can be integrated into a mobile RAN (MNO) to provide local services without considering continuous service with users ${ }^{277}$.

The more advanced prospects for MEC deployments during the integration of MEC frameworks support more services and mobile services based on communication and traffic characteristics ${ }^{278}$. Thus, this MEC-based network could potentially create a heterogeneous computing environment that supports multiple applications ${ }^{279}$.

\section{Conclusion}

MEC provides cloud-based cloud capability, which can support diligent demand, especially the low latency and high bandwidth requirements that arose with $5 \mathrm{G}$ and IoT. With the best proximity, low latency, high performance and more, MEC enables many types of applications. Since MECs play an essential role in $5 \mathrm{G}$ and IoT, we first summarise MEC properties and compare them to the standard MCC in terms of latency, power saving, and background knowledge. we then introduce critical technologies such as cloud services, SDN, NFV, ICN, machine/container devices, smart devices, communication problems, and stream classification to facilitate MEC integration in $5 \mathrm{G}$ and IoT systems. In particular, we analyse the specific components created by MEC and 5G VGDE, providing an overview of MEC capable 5G and IoT applications, such as intelligence - transportation systems, intelligent intelligence, automated business, serious gaming, life healthy, intelligent buildings, smart City AR / VR, intelligent agriculture and intelligent store and organise important part of research when applications for MEC $5 \mathrm{~g}$ and intraocular pressure, coordinator.

We also discuss issues such as edge intelligence, green MEC, MEC server and system installation, mobility management, pricing, security, and privacy. In the end, we recommend using a case using the MEC to implement the knowledge level of IoT. 5G is building a whole new world of services that can be

\footnotetext{
${ }^{273}$ Soatti et al., 2019

${ }^{274}$ Al-Turjman et al., 2018

${ }^{275}$ Al-Turjman et al., 2018

${ }^{276}$ Wang et al., 2018
}

delivered to the business network. Demonstrations such as network integration allow operators to create "shared connections" through virtualisation. Small latency and communication tools allow operators to build books for a wide range of market segments, including healthcare, retail, entertainment, urban intelligence and more. Initially, the goal of multiaccess edge computing was to reduce the cost of bandwidth on the Internet. The emergence of realtime use needs and local data consolidation will boost technology development in the coming years

\section{Reference}

1. Adeel, A., Ahmad, J. and Hussain, A., 2018.

2. Ahmed, S., Rashid, M., Alam, F. and Fakhruddin, B., 2019, November.

3. Alsaeedy and Chong., 2019.

4. Al-Turjman, F., Ever, E. and Zahmatkesh, H., 2018.

5. Asif, AR, Zahra, F. and Matin, MA, 2020.

6. Chen, X., Liu, S., Lu, J., Fan, P. and Letaief, KB, 2016.

7. Chu, E., Yoon, J. and Jung, BC, 2019.

8. Condoluci, M., Lema, MA, Mahmoodi, T. and Dohler, M., 2018.

9. Dai, C., Liu, X., Lai, J., Li, P. and Chao, HC, 2019.

10. Geller, M. and Nair, P., 2018.

11. Hayes, M. and Omar, T., 2019, November.

12. Hossain, MS and Muhammad, G., 2017.

13. Javaid, N., Sher, A., Nasir, H. and Guizani, N., 2018.

14. Lin, BSP, Lin, FJ and Tung, LP, 2016.

15. Luján, E., Mellino, JAZ, Otero, AD, Vega, LR, Galarza, CG and Mocskos, EE, 2019.

16. Mohammadkarimi, M., Dobre, OA and Victory, MZ, 2020.

17. Mohanta, B., Das, P. and Patnaik, S., 2019, May.

18. Monge, MAS, Fernández, BL, Vidal, DM, García, GR, González, AH ma Vidal, JM, 2018, September.

19. Nieto, A., Acien, A. and Fernandez, G., 2019.

20. Rashid, S. and Abd Razak, S., 2019, July.

21. Rezvy, S., Luo, Y., Petridis, M., Lasebae, A. and Zebin, T., 2019, March.

22. Sharma, S., Satapathy, S., Singh, S., Sahu, AK, Obaidat, MS, Saxena, S. and Puthal, D., 2018, December.

23. Sheikh, JA, Akhter, S., Parah, SA and Bhat, GM, 2018.

24. Shin, S. and Kwon, T., 2018.

25. Siddiqi, MA, Yu, H. and Joung, J., 2019.

26. Sk, A. and Masilamani, V., 2018.

\footnotetext{
${ }^{277}$ Shin ma Kwon., 2018

${ }^{278}$ Mohammadkarimi et al., 2020

${ }^{279}$ Stanley et al., 2018
} 
27. Soatti, G., Savazzi, S., Nicoli, M., Alvarez, MA, Kianoush, S., Rampa, V. and Spagnolini, U., 2019.

28. Sodhro, AH, Pirbhulal, S., Sangaiah, AK, Lozano, S., Sodhro, GH and Luo, Z., 5.

29. Stanley, U., North, N., Olu, VM and BobManuel, I., 2018.

30. Trakadas, P., Nomikos, N., Michailidis, ET, Zahariadis, T., Facca, FM, Breitgand, D., Rizzo, S., Masip, X. and Gkonis, P., 2019.

31. Velev, D., Zlateva, P. and Zong, X., 2018, March.

32. Vilalta, R., López, V., Giorgetti, A., Peng, S., Orsini, V., Velasco, L., Serral-Gracia, R., Morris, D., De Fina, S., Cugini, F. and Castoldi, P., 2017.

33. Vukobratovic, D., Jakovetic, D., Skachek, V., Bajovic, D., Sejdinovic, D., Kurt, GK, Hollanti, C. and Fischer, I., 2016.

34. Wang, N., Jiao, L., Wang, P., Dabaghchian, M. and Zeng, K., 2018, December.

35. Zafeiropoulos, A., Fotopoulou, E., Peuster, M., Schneider, S., Gouvas, P., Behnke, D., Müller, M., Bök, PB, Trakadas, P., Karkazis, P. and Karl, H., 2020, June.

36. Zikria, YB, Kim, SW, Afzal, MK, Wang, H. and Rehmani, MH, 2018.

\section{Author's Details}

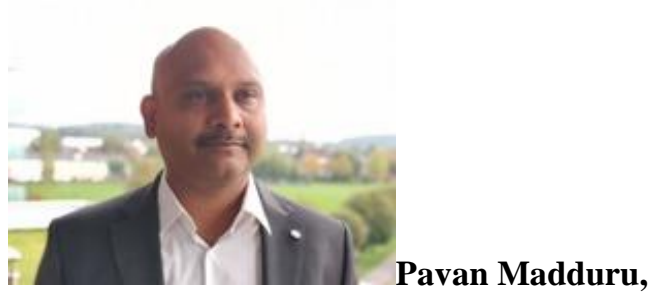

is having around 20 years of experience technology industry experience with 10 years building products and platforms in Artificial intelligence technologies. $\mathrm{He}$ has been working in various roles as Solution architect, product manager/product owner to design, build, and launch tech intensity products B2C and B2B customers globally. 